\title{
Uniformly 2-absorbing primary ideals of commutative rings
}

\author{
H. Mostafanasab, Ü. Tekir, and G. Ulucak
}

\author{
Communicated by V. A. Artamonov
}

\begin{abstract}
A BSTRACT . In this study, we introduce the concept of "uniformly 2-absorbing primary ideals" of commutative rings, which imposes a certain boundedness condition on the usual notion of 2 -absorbing primary ideals of commutative rings. Then we investigate some properties of uniformly 2-absorbing primary ideals of commutative rings with examples. Also, we investigate a specific kind of uniformly 2-absorbing primary ideals by the name of "special 2-absorbing primary ideals".
\end{abstract}

\section{Introduction}

Throughout this paper, we assume that all rings are commutative with $1 \neq 0$. Let $R$ be a commutative ring. An ideal $I$ of $R$ is a proper ideal if $I \neq R$. Then $Z_{I}(R)=\{r \in R \mid r s \in I$ for some $s \in R \backslash I\}$ for a proper ideal $I$ of $R$. Additively, if $I$ is an ideal of $R$, then the radical of $I$ is given by $\sqrt{I}=\left\{r \in R \mid r^{n} \in I\right.$ for some positive integer $\left.n\right\}$. Let $I, J$ be two ideals of $R$. We will denote by $\left(I:_{R} J\right)$, the set of all $r \in R$ such that $r J \subseteq I$.

Cox and Hetzel have introduced uniformly primary ideals of a commutative ring with nonzero identity in [6]. They said that a proper ideal $Q$ of a commutative ring $R$ is uniformly primary if there exists a positive integer $n$ such that whenever $r, s \in R$ satisfy $r s \in Q$ and $r \notin Q$, then

2010 MSC: Primary 13A15; Secondary 13E05, 13F05.

Key words and phrases: uniformly 2-absorbing primary ideal, Noether strongly 2-absorbing primary ideal, 2-absorbing primary ideal. 
$s^{n} \in Q$. A uniformly primary ideal $Q$ has order $N$ and write $\operatorname{ord}_{R}(Q)=N$, or simply $\operatorname{ord}(Q)=N$ if the ring $R$ is understood, if $N$ is the smallest positive integer for which the aforementioned property holds.

Badawi [3] said that a proper ideal $I$ of $R$ is a 2-absorbing ideal of $R$ if whenever $a, b, c \in R$ and $a b c \in I$, then $a b \in I$ or $a c \in I$ or $b c \in I$. He proved that $I$ is a 2-absorbing ideal of $R$ if and only if whenever $I_{1}, I_{2}, I_{3}$ are ideals of $R$ with $I_{1} I_{2} I_{3} \subseteq I$, then $I_{1} I_{2} \subseteq I$ or $I_{1} I_{3} \subseteq I$ or $I_{2} I_{3} \subseteq I$. Anderson and Badawi [1] generalized the notion of 2-absorbing ideals to $n$-absorbing ideals. A proper ideal $I$ of $R$ is called an $n$-absorbing (resp. $a$ strongly $n$-absorbing) ideal if whenever $x_{1} \cdots x_{n+1} \in I$ for $x_{1}, \ldots, x_{n+1} \in R$ (resp. $I_{1} \cdots I_{n+1} \subseteq I$ for ideals $I_{1}, \ldots, I_{n+1}$ of $R$ ), then there are $n$ of the $x_{i}$ 's (resp. $n$ of the $I_{i}$ 's) whose product is in $I$. Badawi et. al. [4] defined a proper ideal $I$ of $R$ to be a 2-absorbing primary ideal of $R$ if whenever $a, b, c \in R$ and $a b c \in I$, then either $a b \in I$ or $a c \in \sqrt{I}$ or $b c \in \sqrt{I}$. Let $I$ be a 2-absorbing primary ideal of $R$. Then $P=\sqrt{I}$ is a 2-absorbing ideal of $R$ by [4, Theorem 2.2]. We say that $I$ is a $P$-2-absorbing primary ideal of $R$. For more studies concerning 2-absorbing (submodules) ideals we refer to $[5,9,10,15,16]$. These concepts motivate us to introduce a generalization of uniformly primary ideals. A proper ideal $Q$ of $R$ is said to be a uniformly 2-absorbing primary ideal of $R$ if there exists a positive integer $n$ such that whenever $a, b, c \in R$ satisfy $a b c \in Q, a b \notin Q$ and $a c \notin \sqrt{Q}$, then $(b c)^{n} \in Q$. In particular, if for $n=1$ the above property holds, then we say that $Q$ is a special 2-absorbing primary ideal of $R$.

In section 2 , we introduce the concepts of uniformly 2-absorbing primary ideals and Noether strongly 2-absorbing primary ideals. Then we investigate the relationship between uniformly 2-absorbing primary ideals, Noether strongly 2-absorbing primary ideals and 2-absorbing primary ideals. After that, in Theorem 2 we characterize uniformly 2-absorbing primary ideals. We show that if $Q_{1}, Q_{2}$ are uniformly primary ideals of a ring $R$, then $Q_{1} \cap Q_{2}$ and $Q_{1} Q_{2}$ are uniformly 2-absorbing primary ideals of $R$, Theorem 4. Let $R=R_{1} \times R_{2}$, where $R_{1}$ and $R_{2}$ are rings with $1 \neq 0$. It is shown (Theorem 5) that a proper ideal $Q$ of $R$ is a uniformly 2-absorbing primary ideal of $R$ if and only if either $Q=Q_{1} \times R_{2}$ for some uniformly 2-absorbing primary ideal $Q_{1}$ of $R_{1}$ or $Q=R_{1} \times Q_{2}$ for some uniformly 2-absorbing primary ideal $Q_{2}$ of $R_{2}$ or $Q=Q_{1} \times Q_{2}$ for some uniformly primary ideal $Q_{1}$ of $R_{1}$ and some uniformly primary ideal $Q_{2}$ of $R_{2}$.

In section 3 , we give some properties of special 2-absorbing primary ideals. For example, in Theorem 7 we show that $Q$ is a special 2-absorbing primary ideal of $R$ if and only if for every ideals $I, J, K$ of $R, I J K \subseteq Q$ implies that either $I J \subseteq \sqrt{Q}$ or $I K \subseteq Q$ or $J K \subseteq Q$. We prove that 
if $Q$ is a special 2-absorbing primary ideal of $R$ and $x \in R \backslash \sqrt{Q}$, then $\left(Q:_{R} x\right)$ is a special 2-absorbing primary ideal of $R$, Theorem 8 . It is proved (Theorem 9) that an irreducible ideal $Q$ of $R$ is special 2-absorbing primary if and only if $\left(Q:_{R} x\right)=\left(Q:_{R} x^{2}\right)$ for every $x \in R \backslash \sqrt{Q}$. Let $R$ be a Prüfer domain and $I$ be an ideal of $R$. In Corollary 10 we show that $Q$ is a special 2-absorbing primary ideal of $R$ if and only if $Q[X]$ is a special 2-absorbing primary ideal of $R[X]$.

\section{Uniformly 2-absorbing primary ideals}

Let $Q$ be a $P$-primary ideal of $R$. We recall from [6] that $Q$ is a Noether strongly primary ideal of $R$ if $P^{n} \subseteq Q$ for some positive integer $n$. We say that $N$ is the exponent of $Q$ if $N$ is the smallest positive integer for which the above property holds and it is denoted by $\mathfrak{e}(Q)=N$.

Definition 1. Let $Q$ be a proper ideal of a ring $R$.

1) $Q$ is a uniformly 2-absorbing primary ideal of $R$ if there exists a positive integer $n$ such that whenever $a, b, c \in R$ satisfy $a b c \in Q$, $a b \notin Q$ and $a c \notin \sqrt{Q}$, then $(b c)^{n} \in Q$. We call that $N$ is order of $Q$ if $N$ is the smallest positive integer for which the above property holds and it is denoted by $2-\operatorname{ord}_{R}(Q)=N$ or $2-\operatorname{ord}(Q)=N$.

2) $P$-2-absorbing primary ideal $Q$ is a Noether strongly 2-absorbing primary ideal of $R$ if $P^{n} \subseteq Q$ for some positive integer $n$. We say that $N$ is the exponent of $Q$ if $N$ is the smallest positive integer for which the above property holds and it is denoted by $2-\mathfrak{e}(Q)=N$.

A valuation ring is an integral domain $V$ such that for every element $x$ of its field of fractions $K$, at least one of $x$ or $x^{-1}$ belongs to $K$.

Proposition 1. Let $V$ be a valuation ring with the quotient field $K$ and let $Q$ be a proper ideal of $V$. The following conditions are equivalent:

1) $Q$ is a uniformly 2-absorbing primary ideal of $V$;

2) There exists a positive integer $n$ such that for every $x, y, z \in K$ whenever $x y z \in Q$ and $x y \notin Q$, then $x z \in \sqrt{Q}$ or $(y z)^{n} \in Q$.

Proof. (1) $\Rightarrow(2)$ Assume that $Q$ is a uniformly 2-absorbing primary ideal of $V$. Let $x y z \in Q$ for some $x, y, z \in K$ such that $x y \notin Q$. If $z \notin V$, then $z^{-1} \in V$, since $V$ is valuation. So $x y z z^{-1}=x y \in Q$, a contradiction. Hence $z \in V$. If $x, y \in V$, then there is nothing to prove. If $y \notin V$, then $x z \in Q \subseteq \sqrt{Q}$, and if $x \notin V$, then $y z \in Q$. Consequently we have the claim.

$(2) \Rightarrow(1)$ It is clear. 
Proposition 2. Let $Q_{1}, Q_{2}$ be two Noether strongly primary ideals of a ring $R$. Then $Q_{1} \cap Q_{2}$ and $Q_{1} Q_{2}$ are Noether strongly 2-absorbing primary ideals of $R$ such that $2-\mathfrak{e}\left(Q_{1} \cap Q_{2}\right) \leqslant \max \left\{\mathfrak{e}\left(Q_{1}\right), \mathfrak{e}\left(Q_{2}\right)\right\}$ and 2-e $\left(Q_{1} Q_{2}\right) \leqslant \mathfrak{e}\left(Q_{1}\right)+\mathfrak{e}\left(Q_{2}\right)$.

Proof. Since $Q_{1}, Q_{2}$ are primary ideals of $R$, then $Q_{1} \cap Q_{2}$ and $Q_{1} Q_{2}$ are 2-absorbing primary ideals of $R$, by [4, Theorem 2.4].

Proposition 3. If $Q$ is a uniformly 2-absorbing primary ideal of $R$, then $Q$ is a 2-absorbing primary ideal of $R$.

Proof. Straightforward.

Proposition 4. Let $R$ be a ring and $Q$ be a proper ideal of $R$.

1) If $Q$ is a 2-absorbing ideal of $R$, then

(a) $Q$ is a Noether strongly 2-absorbing primary ideal with $2-\mathfrak{e}(Q) \leqslant 2$.

(b) $Q$ is a uniformly 2-absorbing primary ideal with 2-ord $(Q)=1$.

2) If $Q$ is a uniformly primary ideal of $R$, then it is a uniformly 2absorbing primary ideal with 2 -ord $(Q)=1$.

Proof. (1) (a) If $Q$ is a 2-absorbing ideal, then it is a 2-absorbing primary ideal and $(\sqrt{Q})^{2} \subseteq Q$, by [3, Theorem 2.4].

(b) It is evident.

(2) Let $Q$ be a uniformly primary ideal of $R$ and let $a b c \in Q$ for some $a, b, c \in R$ such that $a c \notin \sqrt{Q}$. Since $Q$ is uniformly primary, $a b c \in Q$ and $a c \notin \sqrt{Q}$, then $b \in Q$. Therefore $a b \in Q$ or $b c \in Q$. Consequently $Q$ is a uniformly 2-absorbing primary ideal with 2 -ord $(Q)=1$.

Example 1. Let $R=K[X, Y]$ where $K$ is a field. Then $Q=$ $\left(X^{2}, X Y, Y^{2}\right) R$ is a Noether strongly $(X, Y) R$-primary ideal of $R$ and so it is a Noether strongly 2-absorbing primary ideal of $R$.

Proposition 5. If $Q$ is a Noether strongly 2-absorbing primary ideal of $R$, then $Q$ is a uniformly 2-absorbing primary ideal of $R$ and $2-\operatorname{ord}(Q) \leqslant 2$ $\mathfrak{e}(Q)$.

Proof. Let $Q$ be a Noether strongly 2-absorbing primary ideal of $R$. Now, let $a, b, c \in R$ such that $a b c \in Q, a b \notin Q, a c \notin \sqrt{Q}$. Then $b c \in \sqrt{Q}$ since $Q$ is a 2-absorbing primary ideal of $R$. Thus $(b c)^{2-\mathfrak{e}(Q)} \in(\sqrt{Q})^{2-\mathfrak{e}(Q)} \subseteq Q$. Therefore, $Q$ is a uniformly 2-absorbing primary ideal and also 2 - ord $(Q) \leqslant$ $2-\mathfrak{e}(Q)$. 
In the following example, we show that the converse of Proposition 5 is not true. We make use of [6, Example 6 and Example 7]

Example 2. Let $R$ be a ring of characteristic 2 and $T=R[X]$ where $X=$ $\left\{X_{1}, X_{2}, X_{3}, \ldots\right\}$ is a set of indeterminates over $R$. Let $Q=\left(\left\{X_{i}^{2}\right\}_{i=1}^{\infty}\right) T$. By [6, Example 7] $Q$ is a uniformly $P$-primary ideal of $T$ with $\operatorname{ord}_{T}(Q)=1$ where $P=(X) T$. Then $Q$ is a uniformly 2-absorbing primary ideal of $T$ with $2-\operatorname{ord}_{T}(Q)=1$, by Proposition 4(2). But $Q$ is not a Noether strongly 2-absorbing primary ideal since for every positive integer $n, P^{n} \nsubseteq Q$.

Remark 1. Every 2-absorbing ideal of a ring $R$ is a uniformly 2-absorbing primary ideal, but the converse does not necessarily hold. For example, let $p, q$ be two distinct prime numbers. Then $p^{2} q \mathbb{Z}$ is a 2-absorbing primary ideal of $\mathbb{Z},\left[4\right.$, Corollary 2.12]. On the other hand $\left(\sqrt{p^{2} q \mathbb{Z}}\right)^{2}=p^{2} q^{2} \mathbb{Z} \subseteq$ $p^{2} q \mathbb{Z}$, and so $p^{2} q \mathbb{Z}$ is a Noether strongly 2 -absorbing primary ideal of $\mathbb{Z}$. Hence Proposition 5 implies that $p^{2} q \mathbb{Z}$ is a uniformly 2-absorbing primary ideal. But, notice that $p^{2} q \in p^{2} q \mathbb{Z}$ and neither $p^{2} \in p^{2} q \mathbb{Z}$ nor $p q \in p^{2} q \mathbb{Z}$ which shows that $p^{2} q \mathbb{Z}$ is not a 2 -absorbing ideal of $\mathbb{Z}$. Also, it is easy to see that $p^{2} q \mathbb{Z}$ is not primary and so it is not a uniformly primary ideal of $\mathbb{Z}$. Consequently the two concepts of uniformly primary ideals and of uniformly 2-absorbing primary ideals are different in general.

Proposition 6. Let $R$ be a ring and $Q$ be a proper ideal of $R$. If $Q$ is a uniformly 2-absorbing primary ideal of $R$, then one of the following conditions must hold:

1) $\sqrt{Q}=\mathfrak{p}$ is a prime ideal.

2) $\sqrt{Q}=\mathfrak{p} \cap \mathfrak{q}$, where $\mathfrak{p}$ and $\mathfrak{q}$ are the only distinct prime ideals of $R$ that are minimal over $Q$.

Proof. Use [4, Theorem 2.3].

Let $R$ be a ring and $I$ be an ideal of $R$. We denote by $I^{[n]}$ the ideal of $R$ generated by the $n$-th powers of all elements of $I$. If $n$ ! is a unit in $R$, then $I^{[n]}=I^{n}$, see [2].

Theorem 1. Let $Q$ be a proper ideal of $R$. Then the following conditions are equivalent:

1) $Q$ is uniformly primary;

2) There exists a positive integer $n$ such that for every ideals $I, J$ of $R$, $I J \subseteq Q$ implies that either $I \subseteq Q$ or $J^{[n]} \subseteq Q$;

3) There exists a positive integer $n$ such that for every $a \in R$ either $a \in Q$ or $\left(Q:_{R} a\right)^{[n]} \subseteq Q$; 
4) There exists a positive integer $n$ such that for every $a \in R$ either $a^{n} \in Q$ or $(Q: R a)=Q$.

Proof. $(1) \Rightarrow(2)$ Suppose that $Q$ is uniformly primary with $\operatorname{ord}(Q)=n$. Let $I J \subseteq Q$ for some ideals $I, J$ of $R$. Assume that neither $I \subseteq Q$ nor $J^{[n]} \subseteq Q$. Then there exist elements $a \in I \backslash Q$ and $b^{n} \in J^{[n]} \backslash Q$, where $b \in J$. Since $a b \in I J \subseteq Q$, then either $a \in Q$ or $b^{n} \in Q$, which is a contradiction. Therefore either $I \subseteq Q$ or $J^{[n]} \subseteq Q$.

$(2) \Rightarrow(3)$ Note that $a\left(Q:_{R} a\right) \subseteq Q$ for every $a \in R$.

$(3) \Rightarrow(1)$ and $(1) \Leftrightarrow(4)$ have easy verifications.

Corollary 1. Let $R$ be a ring. Suppose that $n$ ! is a unit in $R$ for every positive integer $n$, and $Q$ is a proper ideal of $R$. The following conditions are equivalent:

1) $Q$ is uniformly primary;

2) There exists a positive integer $n$ such that for every ideals $I, J$ of $R$, $I J \subseteq Q$ implies that either $I \subseteq Q$ or $J^{n} \subseteq Q$;

3) There exists a positive integer $n$ such that for every $a \in R$ either $a \in Q$ or $(Q: R a)^{n} \subseteq Q ;$

4) There exists a positive integer $n$ such that for every $a \in R$ either $a^{n} \in Q$ or $\left(Q:_{R} a\right)=Q$.

In the following theorem we characterize uniformly 2-absorbing primary ideals.

Theorem 2. Let $Q$ be a proper ideal of $R$. Then the following conditions are equivalent:

1) $Q$ is uniformly 2-absorbing primary;

2) There exists a positive integer $n$ such that for every $a, b \in R$ either $(a b)^{n} \in Q$ or $\left(Q:_{R} a b\right) \subseteq\left(Q:_{R} a\right) \cup\left(\sqrt{Q}:_{R} b\right) ;$

3) There exists a positive integer $n$ such that for every $a, b \in R$ either $(a b)^{n} \in Q$ or $\left(Q:_{R} a b\right)=\left(Q:_{R} a\right)$ or $\left(Q:_{R} a b\right) \subseteq\left(\sqrt{Q}:_{R} b\right) ;$

4) There exists a positive integer $n$ such that for every $a, b \in R$ and every ideal $I$ of $R, a b I \subseteq Q$ implies that either $a I \subseteq Q$ or $b I \subseteq \sqrt{Q}$ or $(a b)^{n} \in Q$;

5) There exists a positive integer $n$ such that for every $a, b \in R$ either $a b \in Q$ or $\left(Q:_{R} a b\right)^{[n]} \subseteq\left(\sqrt{Q}:_{R} a\right) \cup\left(Q:_{R} b^{n}\right) ;$

6) There exists a positive integer $n$ such that for every $a, b \in R$ either $a b \in Q$ or $\left(Q:_{R} a b\right)^{[n]} \subseteq\left(\sqrt{Q}:_{R} a\right)$ or $\left(Q:_{R} a b\right)^{[n]} \subseteq\left(Q:_{R} b^{n}\right)$.

Proof. $(1) \Rightarrow(2)$ Suppose that $Q$ is uniformly 2-absorbing primary with $2-\operatorname{ord}(Q)=n$. Assume that $a, b \in R$ such that $(a b)^{n} \notin Q$. Let $x \in\left(Q:{ }_{R} a b\right)$. 
Thus $x a b \in Q$, and so either $x a \in Q$ or $x b \in \sqrt{Q}$. Hence $x \in\left(Q:_{R} a\right)$ or $x \in\left(\sqrt{Q}:_{R} b\right)$ which shows that $\left(Q:_{R} a b\right) \subseteq\left(Q:_{R} a\right) \cup\left(\sqrt{Q}:_{R} b\right)$.

$(2) \Rightarrow(3)$ By the fact that if an ideal is a subset of the union of two ideals, then it is a subset of one of them.

$(3) \Rightarrow(4)$ Suppose that $n$ is a positive number which exists by part (3). Let $a, b \in R$ and $I$ be an ideal of $R$ such that $a b I \subseteq Q$ and $(a b)^{n} \notin Q$. Then $I \subseteq\left(Q:_{R} a b\right)$, and so $I \subseteq\left(Q:_{R} a\right)$ or $I \subseteq\left(\sqrt{Q}:_{R} b\right)$, by (3). Consequently $a I \subseteq Q$ or $b I \subseteq \sqrt{Q}$.

$(4) \Rightarrow(1)$ Is easy.

$(1) \Rightarrow(5)$ Suppose that $Q$ is uniformly 2-absorbing primary with 2 - $\operatorname{ord}(Q)=n$. Assume that $a, b \in R$ such that $a b \notin Q$. Let $x \in\left(Q:_{R} a b\right)$. Then $a b x \in Q$. So $a x \in \sqrt{Q}$ or $(b x)^{n} \in Q$. Hence $x^{n} \in\left(\sqrt{Q}:_{R} a\right)$ or $x^{n} \in\left(Q:_{R} b^{n}\right)$. Consequently $\left(Q:_{R} a b\right)^{[n]} \subseteq\left(\sqrt{Q}:_{R} a\right) \cup\left(Q:_{R} b^{n}\right)$.

$(5) \Rightarrow(6)$ Is similar to the proof of $(2) \Rightarrow(3)$.

$(6) \Rightarrow(1)$ Assume (6). Let $a b c \in Q$ for some $a, b, c \in R$ such that $a b \notin Q$. Then $c \in\left(Q:_{R} a b\right)$ and thus $c^{n} \in\left(Q:_{R} a b\right)^{[n]}$. So, by part (6) we have that $c^{n} \in\left(\sqrt{Q}:_{R} a\right)$ or $c^{n} \in\left(Q:_{R} b^{n}\right)$. Therefore $a c \in \sqrt{Q}$ or $(b c)^{n} \in Q$, and so $Q$ is uniformly 2-absorbing primary.

Corollary 2. Let $R$ be a ring. Suppose that $n$ ! is a unit in $R$ for every positive integer $n$, and $Q$ is a proper ideal of $R$. The following conditions are equivalent:

1) $Q$ is uniformly 2-absorbing primary;

2) There exists a positive integer $n$ such that for every $a, b \in R$ either $a b \in Q$ or $\left(Q:_{R} a b\right)^{n} \subseteq\left(\sqrt{Q}:_{R} a\right) \cup\left(Q:_{R} b^{n}\right)$;

3) There exists a positive integer $n$ such that for every $a, b \in R$ either $a b \in Q$ or $\left(Q:_{R} a b\right)^{n} \subseteq\left(\sqrt{Q}:_{R} a\right)$ or $\left(Q:_{R} a b\right)^{n} \subseteq\left(Q:_{R} b^{n}\right)$.

Proposition 7. Let $Q$ be a uniformly 2-absorbing primary ideal of $R$ and $x \in R \backslash Q$ be idempotent. The following conditions hold:

1) $\left(\sqrt{Q}:_{R} x\right)=\sqrt{\left(Q:_{R} x\right)}$.

2) $\left(Q:_{R} x\right)$ is a uniformly 2-absorbing primary ideal of $R$ with 2$\operatorname{ord}((Q: R x)) \leqslant 2-\operatorname{ord}(Q)$.

Proof. (1) Is easy.

(2) Suppose that 2-ord $(Q)=n$. Let $a b c \in\left(Q:_{R} x\right)$ for some $a, b, c \in R$. Then $a(b c) x \in Q$ and so either $a b c \in Q$ or $a x \in \sqrt{Q}$ or $(b c)^{n} x \in Q$. If $a b c \in Q$, then either $a b \in Q \subseteq\left(Q:_{R} x\right)$ or $a c \in \sqrt{Q} \subseteq \sqrt{\left(Q:_{R} x\right)}$ or $(b c)^{n} \in Q \subseteq\left(Q:_{R} x\right)$. If $a x \in \sqrt{Q}$, then $a c \in\left(\sqrt{Q}:_{R} x\right)=\sqrt{\left(Q:_{R} x\right)}$ by part (1). In the third case we have $(b c)^{n} \in\left(Q:_{R} x\right)$. Hence $\left(Q:_{R} x\right)$ is a uniformly 2-absorbing primary ideal of $R$ with 2 -ord $\left(\left(Q:_{R} x\right)\right) \leqslant n$. 
Proposition 8. Let $I$ be a proper ideal of a ring $R$.

1) $\sqrt{I}$ is a 2-absorbing ideal of $R$.

2) For every $a, b, c \in R, a b c \in I$ implies that $a b \in \sqrt{I}$ or $a c \in \sqrt{I}$ or $b c \in \sqrt{I}$;

3) $\sqrt{I}$ is a 2-absorbing primary ideal of $R$;

4) $\sqrt{I}$ is a Noether 2-absorbing primary ideal of $R(2-\mathfrak{e}(\sqrt{I})=1)$;

5) $\sqrt{I}$ is a uniformly 2-absorbing primary ideal of $R$.

Proof. $(1) \Rightarrow(2)$ It is trivial.

$(2) \Rightarrow(1)$ Let $x y z \in \sqrt{I}$ for some $x, y, z \in R$. Then there exists a positive integer $m$ such that $x^{m} y^{m} z^{m} \in I$. So, the hypothesis in (2) implies that $x^{m} y^{m} \in \sqrt{I}$ or $x^{m} z^{m} \in \sqrt{I}$ or $y^{m} z^{m} \in \sqrt{I}$. Hence $x y \in \sqrt{I}$ or $x z \in \sqrt{I}$ or $y z \in \sqrt{I}$ which shows that $\sqrt{I}$ is a 2-absorbing ideal.

$(1) \Leftrightarrow(3)$ and $(3) \Rightarrow(4)$ are clear.

$(4) \Rightarrow(5)$ By Proposition 5 .

$(5) \Rightarrow(3)$ It is easy.

Proposition 9. If $Q_{1}$ is a uniformly P-primary ideal of $R$ and $Q_{2}$ is a uniformly $P$-2-absorbing primary ideal of $R$ such that $Q_{1} \subseteq Q_{2}$, then 2- $\operatorname{ord}\left(Q_{2}\right) \leqslant \operatorname{ord}\left(Q_{1}\right)$.

Proof. Let $\operatorname{ord}\left(Q_{1}\right)=m$ and $2-\operatorname{ord}\left(Q_{2}\right)=n$. Then there are $a, b, c \in R$ such that $a b c \in Q_{2}, a b \notin Q_{2}, a c \notin \sqrt{Q_{2}}$ and $(b c)^{n} \in Q_{2}$ but $(b c)^{n-1} \notin Q_{2}$. Thus $b c \in \sqrt{Q_{2}}=\sqrt{Q_{1}}$. Hence $(b c)^{m} \in Q_{1} \subseteq Q_{2}$ by [6, Proposition 8]. Therefore, $n>m-1$ and so $n \geqslant m$.

Theorem 3. Let $R$ be a ring and $\left\{Q_{i}\right\}_{i \in I}$ be a chain of uniformly P-2absorbing primary ideals such that $\max _{i \in I}\left\{2-\operatorname{ord}\left(Q_{i}\right)\right\}=n$, where $n$ is a positive integer. Then $Q=\bigcap_{i \in I} Q_{i}$ is a uniformly $P$-2-absorbing primary ideal of $R$ with $2-\operatorname{ord}(Q) \leqslant n$.

Proof. It is clear that $\sqrt{Q}=\bigcap_{i \in I} \sqrt{Q_{i}}=P$. Let $a, b, c \in R$ such that $a b c \in Q, a b \notin Q$ and $(b c)^{n} \notin Q$. Since $\left\{Q_{i}\right\}_{i \in I}$ is a chain, there exists some $k \in I$ such that $a b \notin Q_{k}$ and $(b c)^{n} \notin Q_{k}$. On the other hand $Q_{k}$ is uniformly 2-absorbing primary with 2 -ord $\left(Q_{k}\right) \leqslant n$, thus $a c \in \sqrt{Q_{i}}=\sqrt{Q}$, and so $Q$ is a uniformly 2 -absorbing primary ideal of $R$ with 2 -ord $(Q) \leqslant n$.

In the following remark, we show that if $Q_{1}$ and $Q_{2}$ are uniformly 2-absorbing primary ideals of $R$, then $Q_{1} \cap Q_{2}$ need not be a uniformly 2-absorbing primary ideal of $R$. 
Remark 2. Let $p, q, r$ be distinct prime numbers. Then $p^{2} q \mathbb{Z}$ and $r \mathbb{Z}$ are uniformly 2-absorbing primary ideals of $\mathbb{Z}$. Notice that $p^{2} q r \in p^{2} q \mathbb{Z} \cap r \mathbb{Z}$

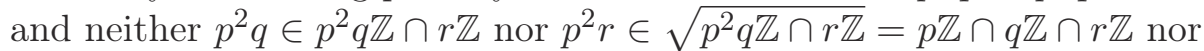
$q r \in \sqrt{p^{2} q \mathbb{Z} \cap r \mathbb{Z}}=p \mathbb{Z} \cap q \mathbb{Z} \cap r \mathbb{Z}$. Hence $p^{2} q \mathbb{Z} \cap r \mathbb{Z}$ is not a 2-absorbing primary ideal of $\mathbb{Z}$ which shows that it is not a uniformly 2-absorbing primary ideal of $\mathbb{Z}$.

Theorem 4. Let $Q_{1}, Q_{2}$ be uniformly primary ideals of a ring $R$.

1) $Q_{1} \cap Q_{2}$ is a uniformly 2-absorbing primary ideal of $R$ with 2$\operatorname{ord}\left(Q_{1} \cap Q_{2}\right) \leqslant \max \left\{\operatorname{ord}\left(Q_{1}\right)\right.$, ord $\left.\left(Q_{2}\right)\right\}$.

2) $Q_{1} Q_{2}$ is a uniformly 2-absorbing primary ideal of $R$ with 2-ord $\left(Q_{1} Q_{2}\right) \leqslant \operatorname{ord}\left(Q_{1}\right)+\operatorname{ord}\left(Q_{2}\right)$.

Proof. (1) Let $Q_{1}, Q_{2}$ be uniformly primary. Set $n=\max \left\{\operatorname{ord}\left(Q_{1}\right)\right.$, $\left.\operatorname{ord}\left(Q_{2}\right)\right\}$. Assume that for some $a, b, c \in R, a b c \in Q_{1} \cap Q_{2}, a b \notin Q_{1} \cap Q_{2}$ and $a c \notin \sqrt{Q_{1} \cap Q_{2}}$. Since $Q_{1}$ and $Q_{2}$ are primary ideals of $R$, then $Q_{1} \cap Q_{2}$ is 2-absorbing primary by [4, Theorem 2.4]. Therefore $b c \in \sqrt{Q_{1} \cap Q_{2}}=$ $\sqrt{Q_{1}} \cap \sqrt{Q_{2}}$. By [6, Proposition 8] we have that $(b c)^{\operatorname{ord}\left(Q_{1}\right)} \in Q_{1}$ and $(b c)^{\operatorname{ord}\left(Q_{2}\right)} \in Q_{2}$. Hence $(b c)^{n} \in Q_{1} \cap Q_{2}$ which shows that $Q_{1} \cap Q_{2}$ is uniformly 2-absorbing primary and 2-ord $\left(Q_{1} \cap Q_{2}\right) \leqslant n$.

(2) Similar to the proof in (1).

We recall from [7], if $R$ is an integral domain and $P$ is a prime ideal of $R$ that can be generated by a regular sequence of $R$, then, for each positive integer $n$, the ideal $P^{n}$ is a $P$-primary ideal of $R$.

Lemma 1. ([6, Corollary 4/) Let $R$ be a ring and $P$ be a prime ideal of $R$. If $P^{n}$ is a P-primary ideal of $R$ for some positive integer $n$, then $P^{n}$ is a uniformly primary ideal of $R$ with $\operatorname{ord}\left(P^{n}\right) \leqslant n$.

Corollary 3. Let $R$ be a ring and $P_{1}, P_{2}$ be prime ideals of $R$. If $P_{1}^{n}$ is a $P_{1}$-primary ideal of $R$ for some positive integer $n$ and $P_{2}^{m}$ is a $P_{2}$-primary ideal of $R$ for some positive integer $m$, then $P_{1}^{n} P_{2}^{m}$ and $P_{1}^{n} \cap P_{2}^{m}$ are uniformly 2-absorbing primary ideals of $R$ with 2-ord $\left(P_{1}^{n} P_{2}^{m}\right) \leqslant n+m$ and 2-ord $\left(P_{1}^{n} \cap P_{2}^{m}\right) \leqslant \max \{n, m\}$.

Proof. By Theorem 4 and Lemma 1.

Proposition 10. Let $f: R \longrightarrow R^{\prime}$ be a homomorphism of commutative rings. Then the following statements hold:

1) If $Q^{\prime}$ is a uniformly 2-absorbing primary ideal of $R^{\prime}$, then $f^{-1}\left(Q^{\prime}\right)$ is a uniformly 2-absorbing primary ideal of $R$ with 2 - $\operatorname{ord}_{R}\left(f^{-1}\left(Q^{\prime}\right)\right) \leqslant$ 2- $\operatorname{ord}_{R^{\prime}}\left(Q^{\prime}\right)$. 
2) If $f$ is an epimorphism and $Q$ is a uniformly 2-absorbing primary ideal of $R$ containing $\operatorname{ker}(f)$, then $f(Q)$ is a uniformly 2-absorbing primary ideal of $R^{\prime}$ with $2-\operatorname{ord}_{R^{\prime}}(f(Q)) \leqslant 2-\operatorname{ord}_{R}(Q)$.

Proof. (1) Set $N=2-\operatorname{ord}_{R^{\prime}}\left(Q^{\prime}\right)$. Let $a, b, c \in R$ such that $a b c \in f^{-1}\left(Q^{\prime}\right)$, $a b \notin f^{-1}\left(Q^{\prime}\right)$ and $a c \notin \sqrt{f^{-1}\left(Q^{\prime}\right)}=f^{-1}\left(\sqrt{Q^{\prime}}\right)$. Then $f(a b c)=$ $f(a) f(b) f(c) \in Q^{\prime}, f(a b)=f(a) f(b) \notin Q^{\prime}$ and $f(a c)=f(a) f(c) \notin \sqrt{Q^{\prime}}$. Since $Q^{\prime}$ is a uniformly 2-absorbing primary ideal of $R^{\prime}$, then $f^{N}(b c) \in Q^{\prime}$. Then $f\left((b c)^{N}\right) \in Q^{\prime}$ and so $(b c)^{N} \in f^{-1}\left(Q^{\prime}\right)$. Thus $f^{-1}\left(Q^{\prime}\right)$ is a uniformly 2-absorbing primary ideal of $R$ with 2- $\operatorname{ord}_{R}\left(f^{-1}\left(Q^{\prime}\right)\right) \leqslant N=2-\operatorname{ord}_{R^{\prime}}\left(Q^{\prime}\right)$.

(2) Set $N=2-\operatorname{ord}_{R}(Q)$. Let $a, b, c \in R^{\prime}$ such that $a b c \in f(Q), a b \notin$ $f(Q)$ and $a c \notin \sqrt{f(Q)}$. Since $f$ is an epimorphism, then there exist $x, y, z \in R$ such that $f(x)=a, f(y)=b$ and $f(z)=c$. Then $f(x y z)=a b c$ $\in f(Q), f(x y)=a b \notin f(Q)$ and $f(x z)=a c \notin \sqrt{f(Q)}$. Since $\operatorname{ker}(f) \subseteq Q$, then $x y z \in Q$. Also $x y \notin Q$, and $x z \notin \sqrt{Q}$, since $f(\sqrt{Q}) \subseteq \sqrt{f(Q)}$. Then $(y z)^{N} \in Q$ since $Q$ is a uniformly 2-absorbing primary ideal of $R$. Thus $f\left((y z)^{N}\right)=(f(y) f(z))^{N}=(b c)^{N} \in f(Q)$. Therefore, $f(Q)$ is a uniformly 2-absorbing primary ideal of $R^{\prime}$. Moreover $2-\operatorname{ord}_{R^{\prime}}(f(Q)) \leqslant$ $N=2-\operatorname{ord}_{R}(Q)$.

As an immediate consequence of Proposition 10 we have the following result:

Corollary 4. Let $R$ be a ring and $Q$ be an ideal of $R$.

1) If $R^{\prime}$ is a subring of $R$ and $Q$ is a uniformly 2-absorbing primary ideal of $R$, then $Q \cap R^{\prime}$ is a uniformly 2-absorbing primary ideal of $R^{\prime}$ with $2-\operatorname{ord}_{R^{\prime}}\left(Q \cap R^{\prime}\right) \leqslant 2-\operatorname{ord}_{R}(Q)$.

2) Let $I$ be an ideal of $R$ with $I \subseteq Q$. Then $Q$ is a uniformly 2-absorbing primary ideal of $R$ if and only if $Q / I$ is a uniformly 2-absorbing primary ideal of $R / I$.

Corollary 5. Let $Q$ be an ideal of a ring $R$. Then $\langle Q, X\rangle$ is a uniformly 2absorbing primary ideal of $R[X]$ if and only if $Q$ is a uniformly 2-absorbing primary ideal of $R$.

Proof. By Corollary 4(2) and regarding the isomorphism $\langle Q, X\rangle /\langle X\rangle \simeq Q$ in $R[X] /\langle X\rangle \simeq R$ we have the result.

Corollary 6. Let $R$ be a ring, $Q$ a proper ideal of $R$ and $X=\left\{X_{i}\right\}_{i \in I}$ a collection of indeterminates over $R$. If $Q R[X]$ is a uniformly 2-absorbing primary ideal of $R[X]$, then $Q$ is a uniformly 2-absorbing primary ideal of $R$ with $2-\operatorname{ord}_{R}(Q) \leqslant 2-\operatorname{ord}_{R[X]}(Q R[X])$. 
Proof. It is clear from Corollary 4(1).

Proposition 11. Let $S$ be a multiplicatively closed subset of $R$ and $Q$ be a proper ideal of $R$. Then the following conditions hold:

1) If $Q$ is a uniformly 2-absorbing primary ideal of $R$ such that $Q \cap S=$ $\varnothing$, then $S^{-1} Q$ is a uniformly 2-absorbing primary ideal of $S^{-1} R$ with 2 - $\operatorname{ord}\left(S^{-1} Q\right) \leqslant 2$ - ord $(Q)$.

2) If $S^{-1} Q$ is a uniformly 2-absorbing primary ideal of $S^{-1} R$ and $S \cap Z_{Q}(R)=\varnothing$, then $Q$ is a uniformly 2-absorbing primary ideal of $R$ with 2 - ord $(Q) \leqslant 2-\operatorname{ord}\left(S^{-1} Q\right)$.

Proof. (1) Set $N:=2$ - ord $(Q)$. Let $a, b, c \in R$ and $s, t, k \in S$ such that $\frac{a}{s} \frac{b}{t} \frac{c}{k} \in S^{-1} Q, \frac{a}{s} \frac{b}{t} \notin S^{-1} Q, \frac{a}{s} \frac{c}{k} \notin \sqrt{S^{-1} Q}=S^{-1} \sqrt{Q}$. Thus there is $u \in S$ such that $u a b c \in Q$. By assumptions we have that $u a b \notin Q$ and $u a c \notin \sqrt{Q}$. Since $Q$ is a uniformly 2-absorbing primary ideal of $R$, then $(b c)^{N} \in Q$. Hence $\left(\frac{b}{t} \frac{c}{k}\right)^{N} \in S^{-1} Q$. Consequently, $S^{-1} Q$ is a uniformly 2-absorbing primary ideal of $S^{-1} R$ and $2-\operatorname{ord}\left(S^{-1} Q\right) \leqslant N=2-\operatorname{ord}(Q)$.

(2) Set $N:=2-\operatorname{ord}\left(S^{-1} Q\right)$. Let $a, b, c \in R$ such that $a b c \in Q, a b \notin$ $Q$ and $a c \notin \sqrt{Q}$. Then $\frac{a b c}{1}=\frac{a}{1} \frac{b}{1} \frac{c}{1} \in S^{-1} Q, \frac{a b}{1}=\frac{a}{1} \frac{b}{1} \notin S^{-1} Q$ and $\frac{a c}{1}=\frac{a}{1} \frac{c}{1} \notin \sqrt{S^{-1} Q}=S^{-1} \sqrt{Q}$, because $S \cap Z_{Q}(R)=\varnothing$ and $S \cap Z_{\sqrt{Q}}(R)=$ $\varnothing$. Since $S^{-1} Q$ is a uniformly 2-absorbing primary ideal of $S^{-1} R$, then $\left(\frac{b}{1} \frac{c}{1}\right)^{N}=\frac{(b c)^{N}}{1} \in S^{-1} Q$. Then there exists $u \in S$ such that $u(b c)^{N} \in Q$. Hence $(b c)^{N} \in Q$ because $S \cap Z_{Q}(R)=\varnothing$. Thus $Q$ is a uniformly 2absorbing primary ideal of $R$ and $2-\operatorname{ord}(Q) \leqslant N=2-\operatorname{ord}\left(S^{-1} Q\right)$.

Proposition 12. Let $Q$ be a 2-absorbing primary ideal of a ring $R$ and $P=\sqrt{Q}$ be a finitely generated ideal of $R$. Then $Q$ is a Noether strongly 2-absorbing primary ideal of $R$. Thus $Q$ is a uniformly 2-absorbing primary ideal of $R$.

Proof. It is clear from [14, Lemma 8.21] and Proposition 5.

Corollary 7. Let $R$ be a Noetherian ring and $Q$ a proper ideal of $R$. Then the following conditions are equivalent:

1) $Q$ is a uniformly 2-absorbing primary ideal of $R$;

2) $Q$ is a Noether strongly 2-absorbing primary ideal of $R$;

3) $Q$ is a 2-absorbing primary ideal of $R$.

Proof. Apply Proposition 5 and Proposition 12. 
We recall from [8] the construction of idealization of a module. Let $R$ be a ring and $M$ be an $R$-module. Then $R(+) M=R \times M$ is a ring with identity $(1,0)$ under addition defined by $(r, m)+(s, n)=(r+s, m+n)$ and multiplication defined by $(r, m)(s, n)=(r s, r n+s m)$. Note that $\sqrt{I}(+) M=\sqrt{I(+) M}$.

Proposition 13. Let $R$ be a ring, $Q$ be a proper ideal of $R$ and $M$ be an $R$-module. The following conditions are equivalent:

1) $Q(+) M$ is a uniformly 2-absorbing primary ideal of $R(+) M$;

2) $Q$ is a uniformly 2-absorbing primary ideal of $R$.

Proof. The proof is routine.

Theorem 5. Let $R=R_{1} \times R_{2}$, where $R_{1}$ and $R_{2}$ are rings with $1 \neq 0$. Let $Q$ be a proper ideal of $R$. Then the following conditions are equivalent:

1) $Q$ is a uniformly 2-absorbing primary ideal of $R$;

2) Either $Q=Q_{1} \times R_{2}$ for some uniformly 2-absorbing primary ideal $Q_{1}$ of $R_{1}$ or $Q=R_{1} \times Q_{2}$ for some uniformly 2-absorbing primary ideal $Q_{2}$ of $R_{2}$ or $Q=Q_{1} \times Q_{2}$ for some uniformly primary ideal $Q_{1}$ of $R_{1}$ and some uniformly primary ideal $Q_{2}$ of $R_{2}$.

Proof. (1) $\Rightarrow(2)$ Assume that $Q$ is a uniformly 2-absorbing primary ideal of $R$ with 2-ord $R(Q)=n$. We know that $Q$ is in the form of $Q_{1} \times Q_{2}$ for some ideal $Q_{1}$ of $R_{1}$ and some ideal $Q_{2}$ of $R_{2}$. Suppose that $Q_{2}=R_{2}$. Since $Q$ is a proper ideal of $R, Q_{1} \neq R_{1}$. Let $R^{\prime}=\frac{R}{\{0\} \times R_{2}}$. Then $Q^{\prime}=\frac{Q}{\{0\} \times R_{2}}$ is a uniformly 2-absorbing primary ideal of $R^{\prime}$ by Corollary 4(2). Since $R^{\prime}$ is ring-isomorphic to $R_{1}$ and $Q_{1} \simeq Q^{\prime}, Q_{1}$ is a uniformly 2-absorbing primary ideal of $R_{1}$. Suppose that $Q_{1}=R_{1}$. Since $Q$ is a proper ideal of $R, Q_{2} \neq R_{2}$. By a similar argument as in the previous case, $Q_{2}$ is a uniformly 2-absorbing primary ideal of $R_{2}$. Hence assume that $Q_{1} \neq R_{1}$ and $Q_{2} \neq R_{2}$. We claim that $Q_{1}$ is a uniformly primary ideal of $R_{1}$. Assume that $x, y \in R_{1}$ such that $x y \in Q_{1}$ but $x \notin Q_{1}$. Notice that $(x, 1)(1,0)(y, 1)=(x y, 0) \in Q$, but neither $(x, 1)(1,0)=(x, 0) \in Q$ nor $(x, 1)(y, 1)=(x y, 1) \in \sqrt{Q}$. So $[(1,0)(y, 1)]^{n}=\left(y^{n}, 0\right) \in Q$. Therefore $y^{n} \in Q_{1}$. Thus $Q_{1}$ is a uniformly primary ideal of $R_{1}$ with $\operatorname{ord}_{R_{1}}\left(Q_{1}\right) \leqslant n$. Now, we claim that $Q_{2}$ is a uniformly primary ideal of $R_{2}$. Suppose that for some $z, w \in R_{2}, z w \in Q_{2}$ but $z \notin Q_{2}$. Notice that $(1, z)(0,1)(1, w)=(0, z w) \in Q$, but neither $(1, z)(0,1)=(0, z) \in Q$ nor $(1, z)(1, w)=(1, z w) \in \sqrt{Q}$. Therefore $[(0,1)(1, w)]^{n}=\left(0, w^{n}\right) \in Q$, and so $w^{n} \in Q_{2}$ which shows that $Q_{2}$ is a uniformly primary ideal of $R_{2}$ with $\operatorname{ord}_{R_{2}}\left(Q_{2}\right) \leqslant n$. Consequently 
when $Q_{1} \neq R_{1}$ and $Q_{2} \neq R_{2}$ we have that $\max \left\{\operatorname{ord}_{R_{1}}\left(Q_{1}\right), \operatorname{ord}_{R_{2}}\left(Q_{2}\right)\right\} \leqslant$ $2-\operatorname{ord}_{R}(Q)$.

(2) $\Rightarrow$ (1) If $Q=Q_{1} \times R_{2}$ for some uniformly 2-absorbing primary ideal $Q_{1}$ of $R_{1}$, or $Q=R_{1} \times Q_{2}$ for some uniformly 2-absorbing primary ideal $Q_{2}$ of $R_{2}$, then it is clear that $Q$ is a uniformly 2-absorbing primary ideal of $R$. Hence assume that $Q=Q_{1} \times Q_{2}$ for some uniformly primary ideal $Q_{1}$ of $R_{1}$ and some uniformly primary ideal $Q_{2}$ of $R_{2}$. Then $Q_{1}^{\prime}=$ $Q_{1} \times R_{2}$ and $Q_{2}^{\prime}=R_{1} \times Q_{2}$ are uniformly primary ideals of $R$ with $\operatorname{ord}_{R}\left(Q_{1}^{\prime}\right) \leqslant \operatorname{ord}_{R_{1}}\left(Q_{1}\right)$ and $\operatorname{ord}_{R}\left(Q_{2}^{\prime}\right) \leqslant \operatorname{ord}_{R_{2}}\left(Q_{2}\right)$. Hence $Q_{1}^{\prime} \cap Q_{2}^{\prime}=$ $Q_{1} \times Q_{2}=Q$ is a uniformly 2-absorbing primary ideal of $R$ with 2$\operatorname{ord}_{R}(Q) \leqslant \max \left\{\operatorname{ord}_{R_{1}}\left(Q_{1}\right), \operatorname{ord}_{R_{2}}\left(Q_{2}\right)\right\}$ by Theorem 4 .

Lemma 2. Let $R=R_{1} \times R_{2} \times \cdots \times R_{n}$, where $R_{1}, R_{2}, \ldots, R_{n}$ are rings with $1 \neq 0$. A proper ideal $Q$ of $R$ is a uniformly primary ideal of $R$ if and only if $Q=\times_{i=1}^{n} Q_{i}$ such that for some $k \in\{1,2, \ldots, n\}, Q_{k}$ is a uniformly primary ideal of $R_{k}$, and $Q_{i}=R_{i}$ for every $i \in\{1,2, \ldots, n\} \backslash\{k\}$.

Proof. $(\Rightarrow)$ Let $Q$ be a uniformly primary ideal of $R$ with $\operatorname{ord}_{R}(Q)=m$. We know $Q=\times_{i=1}^{n} Q_{i}$ where for every $1 \leqslant i \leqslant n, Q_{i}$ is an ideal of $R_{i}$, respectively. Assume that $Q_{r}$ is a proper ideal of $R_{r}$ and $Q_{s}$ is a proper ideal of $R_{s}$ for some $1 \leqslant r<s \leqslant n$. Since

$$
(0, \ldots, 0, \overbrace{1_{R_{r}}}^{r \text {-th }}, 0, \ldots, 0)(0, \ldots, 0, \overbrace{1_{R_{s}}}^{s \text {-th }}, 0, \ldots, 0)=(0, \ldots, 0) \in Q,
$$

then either $(0, \ldots, 0, \overbrace{1_{R_{r}}}^{r \text {-th }}, 0, \ldots, 0) \in Q$ or $(0, \ldots, 0, \overbrace{1_{R_{s}}}^{s \text {-th }}, 0, \ldots, 0)^{m} \in Q$, which is a contradiction. Hence exactly one of the $Q_{i}$ 's is proper, say $Q_{k}$. Now, we show that $Q_{k}$ is a uniformly primary ideal of $R_{k}$. Let $a b \in Q_{k}$ for some $a, b \in R_{k}$ such that $a \notin Q_{k}$. Therefore

$$
\begin{gathered}
(0, \ldots, 0, \overbrace{a}^{k \text { th }}, 0, \ldots, 0)(0, \ldots, 0, \overbrace{b}^{k \text {-th }}, 0, \ldots, 0) \\
=(0, \ldots, 0, \overbrace{a b}^{k \text { th }}, 0, \ldots, 0) \in Q,
\end{gathered}
$$

but $(0, \ldots, 0, \overbrace{a}^{k \text { th }}, 0, \ldots, 0) \notin Q$, and so $(0, \ldots, 0, \overbrace{b}^{k \text {-th }}, 0, \ldots, 0)^{m} \in Q$. Thus $b^{m} \in Q_{k}$ which implies that $Q_{k}$ is a uniformly primary ideals of $R_{k}$ with $\operatorname{ord}_{R_{k}}\left(Q_{k}\right) \leqslant m$.

$(\Leftarrow)$ Is easy. 
Theorem 6. Let $R=R_{1} \times R_{2} \times \cdots \times R_{n}$, where $2 \leqslant n<\infty$, and $R_{1}, R_{2}, \ldots, R_{n}$ are rings with $1 \neq 0$. For a proper ideal $Q$ of $R$ the following conditions are equivalent:

1) $Q$ is a uniformly 2-absorbing primary ideal of $R$.

2) Either $Q=\times_{t=1}^{n} Q_{t}$ such that for some $k \in\{1,2, \ldots, n\}, Q_{k}$ is a uniformly 2-absorbing primary ideal of $R_{k}$, and $Q_{t}=R_{t}$ for every $t \in\{1,2, \ldots, n\} \backslash\{k\}$ or $Q=\times_{t=1}^{n} Q_{t}$ such that for some $k, m \in\{1,2, \ldots, n\}, Q_{k}$ is a uniformly primary ideal of $R_{k}, Q_{m}$ is a uniformly primary ideal of $R_{m}$, and $Q_{t}=R_{t}$ for every $t \in$ $\{1,2, \ldots, n\} \backslash\{k, m\}$.

Proof. We use induction on $n$. For $n=2$ the result holds by Theorem 5 . Then let $3 \leqslant n<\infty$ and suppose that the result is valid when $K=R_{1} \times \cdots \times R_{n-1}$. We show that the result holds when $R=K \times R_{n}$. By Theorem 5, $Q$ is a uniformly 2-absorbing primary ideal of $R$ if and only if either $Q=L \times R_{n}$ for some uniformly 2-absorbing primary ideal $L$ of $K$ or $Q=K \times L_{n}$ for some uniformly 2-absorbing primary ideal $L_{n}$ of $R_{n}$ or $Q=L \times L_{n}$ for some uniformly primary ideal $L$ of $K$ and some uniformly primary ideal $L_{n}$ of $R_{n}$. Notice that by Lemma 2, a proper ideal $L$ of $K$ is a uniformly primary ideal of $K$ if and only if $L=\times_{t=1}^{n-1} Q_{t}$ such that for some $k \in\{1,2, \ldots, n-1\}, Q_{k}$ is a uniformly primary ideal of $R_{k}$, and $Q_{t}=R_{t}$ for every $t \in\{1,2, \ldots, n-1\} \backslash\{k\}$. Consequently we reach the claim.

\section{Special 2-absorbing primary ideals}

Definition 2. We say that a proper ideal $Q$ of a ring $R$ is special 2absorbing primary if it is uniformly 2-absorbing primary with 2 -ord $(Q)=1$.

Remark 3. By Proposition 4(2), every primary ideal is a special 2absorbing primary ideal. But the converse is not true in general. For example, let $p, q$ be two distinct prime numbers. Then $p q \mathbb{Z}$ is a 2 -absorbing ideal of $\mathbb{Z}$ and so it is a special 2-absorbing primary ideal of $\mathbb{Z}$, by Proposition $4(1)$. Clearly $p q \mathbb{Z}$ is not primary.

Recall that a prime ideal $\mathfrak{p}$ of $R$ is called divided prime if $\mathfrak{p} \subset x R$ for every $x \in R \backslash \mathfrak{p}$.

Proposition 14. Let $Q$ be a special 2-absorbing primary ideal of $R$ such that $\sqrt{Q}=\mathfrak{p}$ is a divided prime ideal of $R$. Then $Q$ is a $\mathfrak{p}$-primary ideal of $R$. 
Proof. Let $x y \in Q$ for some $x, y \in R$ such that $y \notin \mathfrak{p}$. Then $x \in \mathfrak{p}$. Since $\mathfrak{p}$ is a divided prime ideal, $\mathfrak{p} \subset y R$ and so there exists $r \in R$ such that $x=r y$. Hence $x y=r y^{2} \in Q$. Since $Q$ is special 2-absorbing primary and $y \notin \mathfrak{p}$, then $x=r y \in Q$. Consequently $Q$ is a $\mathfrak{p}$-primary ideal of $R$.

Remark 4. Let $p, q$ be distinct prime numbers. Then by [4, Theorem 2.4 we can deduce that $p \mathbb{Z} \cap q^{2} \mathbb{Z}$ is a 2 -absorbing primary ideal of $\mathbb{Z}$. Since $p q^{2} \in p \mathbb{Z} \cap q^{2} \mathbb{Z}, p q \notin p \mathbb{Z} \cap q^{2} \mathbb{Z}$ and $q^{2} \notin p \mathbb{Z} \cap q \mathbb{Z}$, then $p \mathbb{Z} \cap q^{2} \mathbb{Z}$ is not a special 2-absorbing primary ideal of $\mathbb{Z}$.

Notice that for $n=1$ we have that $I^{[n]}=I$.

Theorem 7. Let $Q$ be a proper ideal of $R$. Then the following conditions are equivalent:

1) $Q$ is special 2-absorbing primary;

2) For every $a, b \in R$ either $a b \in Q$ or $\left(Q:_{R} a b\right)=\left(\begin{array}{lll}Q & :_{R} & a\end{array}\right)$ or $\left(Q:_{R} a b\right) \subseteq\left(\sqrt{Q}:_{R} b\right)$;

3) For every $a, b \in R$ and every ideal $I$ of $R, a b I \subseteq Q$ implies that either $a b \in Q$ or $a I \subseteq Q$ or $b I \subseteq \sqrt{Q}$;

4) For every $a \in R$ and every ideal $I$ of $R$ either $a I \subseteq Q$ or $(Q: R$ $a I) \subseteq\left(Q:_{R} a\right) \cup\left(\sqrt{Q}:_{R} I\right)$;

5) For every $a \in R$ and every ideal $I$ of $R$ either $a I \subseteq Q$ or $(Q: R$ $a I)=\left(Q:_{R} a\right)$ or $\left(Q:_{R} a I\right) \subseteq\left(\sqrt{Q}:_{R} I\right)$;

6) For every $a \in R$ and every ideals $I, J$ of $R, a I J \subseteq Q$ implies that either $a I \subseteq Q$ or $I J \subseteq \sqrt{Q}$ or $a J \subseteq Q$;

7) For every ideals $I, J$ of $R$ either $I J \subseteq \sqrt{Q}$ or $\left(Q:_{R} I J\right) \subseteq\left(Q:_{R}\right.$ $I) \cup(Q: R J)$;

8) For every ideals $I, J$ of $R$ either $I J \subseteq \sqrt{Q}$ or $\left(Q:_{R} I J\right)=\left(Q:_{R} I\right)$ or $\left(Q:_{R} I J\right)=\left(Q:_{R} J\right)$;

9) For every ideals $I, J, K$ of $R, I J K \subseteq Q$ implies that either $I J \subseteq \sqrt{Q}$ or $I K \subseteq Q$ or $J K \subseteq Q$.

Proof. $(1) \Leftrightarrow(2) \Leftrightarrow(3)$ By Theorem 2.

(3) $\Rightarrow(4)$ Let $a \in R$ and $I$ be an ideal of $R$ such that $a I \nsubseteq Q$. Suppose that $x \in\left(Q:_{R} a I\right)$. Then $a x I \subseteq Q$, and so by part (3) we have that $x \in$ $\left(Q:_{R} a\right)$ or $x \in\left(\sqrt{Q}:_{R} I\right)$. Therefore $\left(Q:_{R} a I\right) \subseteq\left(Q:_{R} a\right) \cup\left(\sqrt{Q}:_{R} I\right)$.

$(4) \Rightarrow(5) \Rightarrow(6) \Rightarrow(7) \Rightarrow(8) \Rightarrow(9) \Rightarrow(1)$ Have straightforward proofs.

Theorem 8. Let $Q$ be a special 2-absorbing primary ideal of $R$ and $x \in R \backslash \sqrt{Q}$. The following conditions hold:

1) $\left(Q:_{R} x\right)=\left(Q:_{R} x^{n}\right)$ for every $n \geqslant 2$. 
2) $\left(\sqrt{Q}:_{R} x\right)=\sqrt{\left(Q:_{R} x\right)}$.

3) $(Q: R x)$ is a special 2-absorbing primary ideal of $R$.

Proof. (1) Clearly $\left(Q:_{R} x\right) \subseteq\left(Q:_{R} x^{n}\right)$ for every $n \geqslant 2$. For the converse inclusion we use induction on $n$. First we get $n=2$. Let $r \in\left(Q:_{R} x^{2}\right)$. Then $r x^{2} \in Q$, and so either $r x \in Q$ or $x^{2} \in \sqrt{Q}$. Notice that $x^{2} \in \sqrt{Q}$ implies that $x \in \sqrt{Q}$ which is a contradiction. Therefore $r x \in Q$ and so $r \in\left(Q:_{R} x\right)$. Therefore $\left(Q:_{R} x\right)=\left(Q:_{R} x^{2}\right)$. Now, assume $n>2$ and suppose that the claim holds for $n-1$, i.e. $\left(Q:_{R} x\right)=\left(Q:_{R} x^{n-1}\right)$. Let $r \in\left(Q:_{R} x^{n}\right)$. Then $r x^{n} \in Q$. Since $x \notin \sqrt{Q}$, then we have either $r x^{n-1} \in$ $Q$ or $r x \in Q$. Both two cases implies that $r \in\left(Q:_{R} x\right)$. Consequently $\left(Q:_{R} x\right)=\left(Q:_{R} x^{n}\right)$.

(2) It is easy to investigate that $\sqrt{\left(Q:_{R} x\right)} \subseteq\left(\sqrt{Q}:_{R} x\right)$. Let $r \in$ $\left(\sqrt{Q}:_{R} x\right)$. Then there exists a positive integer $m$ such that $(r x)^{m} \in Q$. So, by part (1) we have that $r^{m} \in\left(Q:_{R} x\right)$. Hence $r \in \sqrt{\left(Q:_{R} x\right)}$. Thus $\left(\sqrt{Q}:_{R} x\right)=\sqrt{\left(Q:_{R} x\right)}$.

(3) Let $a b c \in\left(Q:_{R} x\right)$ for some $a, b, c \in R$. Then $a x(b c) \in Q$ and so $a x \in Q$ or $a b c \in Q$ or $b c x \in \sqrt{Q}$. In the first case, we have $a b \in\left(Q:_{R} x\right)$. If $a b c \in Q$, then either $a b \in Q \subseteq\left(Q:_{R} x\right)$ or $a c \in Q \subseteq\left(Q:_{R} x\right)$ or $b c \in$ $\sqrt{Q} \subseteq \sqrt{\left(Q:_{R} x\right)}$. In the third case we have $b c \in\left(\sqrt{Q}:_{R} x\right)=\sqrt{\left(Q:_{R} x\right)}$ by part (2). Therefore $\left(Q:_{R} x\right)$ is a special 2-absorbing primary ideal of $R$.

Theorem 9. Let $Q$ be an irreducible ideal of $R$. Then $Q$ is special 2absorbing primary if and only if $\left(Q:_{R} x\right)=\left(Q:_{R} x^{2}\right)$ for every $x \in R \backslash \sqrt{Q}$.

Proof. $(\Rightarrow)$ By Theorem 8 .

$(\Leftarrow)$ Let $a b c \in Q$ for some $a, b, c \in R$ such that neither $a b \in Q$ nor $a c \in Q$ nor $b c \in \sqrt{Q}$. We search for a contradiction. Since $b c \notin \sqrt{Q}$, then $b \notin \sqrt{Q}$. So, by our hypothesis we have $\left(Q:_{R} b\right)=\left(Q:_{R} b^{2}\right)$. Let $r \in(Q+R a b) \cap(Q+R a c)$. Then there are $q_{1}, q_{2} \in Q$ and $r_{1}, r_{2} \in R$ such that $r=q_{1}+r_{1} a b=q_{2}+r_{2} a c$. Hence $q_{1} b+r_{1} a b^{2}=q_{2} b+r_{2} a b c \in Q$. Thus $r_{1} a b^{2} \in Q$, i.e., $r_{1} a \in\left(Q:_{R} b^{2}\right)=\left(Q:_{R} b\right)$. Therefore $r_{1} a b \in Q$ and so $r=q_{1}+r_{1} a b \in Q$. Then $Q=(Q+R a b) \cap(Q+R a c)$, which contradicts the assumption that $Q$ is irreducible.

A ring $R$ is said to be a Boolean ring if $x=x^{2}$ for all $x \in R$. It is famous that every prime ideal in a Boolean ring $R$ is maximal. Notice that every ideal of a Boolean ring $R$ is radical. So, every (uniformly) 2-absorbing primary ideal of $R$ is a 2-absorbing ideal of $R$. 
Corollary 8. Let $R$ be a Boolean ring. Then every irreducible ideal of $R$ is a maximal ideal.

Proof. Let $I$ be an irreducible ideal of $R$. Thus, Theorem 9 implies that $I$ is special 2-absorbing primary. Therefore by Proposition 6 , either $I=\sqrt{I}$ is a maximal ideal or is the intersection of two distinct maximal ideals. Since $I$ is irreducible, then $I$ cannot be in the second form. Hence $I$ is a maximal ideal.

Proposition 15. Let $Q$ be a special 2-absorbing primary ideal of $R$ and $\mathfrak{p}, \mathfrak{q}$ be distinct prime ideals of $R$.

1) If $\sqrt{Q}=\mathfrak{p}$, then $\{(Q: R x) \mid x \in R \backslash \mathfrak{p}\}$ is a totally ordered set.

2) If $\sqrt{Q}=\mathfrak{p} \cap \mathfrak{q}$, then $\{(Q: R x) \mid x \in R \backslash \mathfrak{p} \cup \mathfrak{q}\}$ is a totally ordered set.

Proof. (1) Let $x, y \in R \backslash \mathfrak{p}$. Then $x y \in R \backslash \mathfrak{p}$. It is clear that $\left(Q:_{R} x\right) \cup\left(Q:_{R}\right.$ $y) \subseteq\left(Q:_{R} x y\right)$. Assume that $r \in\left(Q:_{R} x y\right)$. Therefore $r x y \in Q$, whence $r x \in Q$ or $r y \in Q$, because $x y \notin \sqrt{Q}$. Consequently $\left(Q:_{R} x y\right)=\left(Q:_{R}\right.$ $x) \cup\left(Q:_{R} y\right)$. Thus, either $\left(Q:_{R} x y\right)=\left(Q:_{R} x\right)$ or $\left(Q:_{R} x y\right)=\left(Q:_{R} y\right)$, and so either $\left(Q:_{R} y\right) \subseteq\left(Q:_{R} x\right)$ or $\left(Q:_{R} x\right) \subseteq\left(Q:_{R} y\right)$.

(2) Is similar to the proof of (1).

Corollary 9. Let $f: R \longrightarrow R^{\prime}$ be a homomorphism of commutative rings. Then the following statements hold:

1) If $Q^{\prime}$ is a special 2-absorbing primary ideal of $R^{\prime}$, then $f^{-1}\left(Q^{\prime}\right)$ is a special 2-absorbing primary ideal of $R$.

2) If $f$ is an epimorphism and $Q$ is a special 2-absorbing primary ideal of $R$ containing $\operatorname{ker}(f)$, then $f(Q)$ is a special 2-absorbing primary ideal of $R^{\prime}$.

Proof. By Proposition 10.

Let $R$ be a ring with identity. We recall that if $f=a_{0}+a_{1} X+\cdots+a_{t} X^{t}$ is a polynomial on the ring $R$, then content of $f$ is defined as the ideal of $R$, generated by the coefficients of $f$, i.e. $c(f)=\left(a_{0}, a_{1}, \ldots, a_{t}\right)$. Let $T$ be an $R$-algebra and $c$ the function from $T$ to the ideals of $R$ defined by $c(f)=\cap\{I \mid I$ is an ideal of $R$ and $f \in I T\}$ known as the content of $f$. Note that the content function $c$ is nothing but the generalization of the content of a polynomial $f \in R[X]$. The $R$-algebra $T$ is called a content $R$-algebra if the following conditions hold:

1) For all $f \in T, f \in c(f) T$. 
2) (Faithful flatness) For any $r \in R$ and $f \in T$, the equation $c(r f)=$ $r c(f)$ holds and $c\left(1_{T}\right)=R$.

3) (Dedekind-Mertens content formula) For each $f, g \in T$, there exists a natural number $n$ such that $c(f)^{n} c(g)=c(f)^{n-1} c(f g)$.

For more information on content algebras and their examples we refer to [11], [12] and [13]. In [10] Nasehpour gave the definition of a Gaussian $R$-algebra as follows: Let $T$ be an $R$-algebra such that $f \in c(f) T$ for all $f \in T$. T is said to be a Gaussian $R$-algebra if $c(f g)=c(f) c(g)$, for all $f, g \in T$.

Example 3. ([10]) Let $T$ be a content $R$-algebra such that $R$ is a Prüfer domain. Since every nonzero finitely generated ideal of $R$ is a cancellation ideal of $R$, the Dedekind-Mertens content formula causes $T$ to be a Gaussian $R$-algebra.

Theorem 10. Let $R$ be a Prüfer domain, $T$ a content $R$-algebra and $Q$ an ideal of $R$. Then $Q$ is a special 2-absorbing primary ideal of $R$ if and only if $Q T$ is a special 2-absorbing primary ideal of $T$.

Proof. $(\Rightarrow)$ Assume that $Q$ is a special 2-absorbing primary ideal of $R$. Let $f g h \in Q T$ for some $f, g, h \in T$. Then $c(f g h) \subseteq Q$. Since $R$ is a Prüfer domain and $T$ is a content $R$-algebra, then $T$ is a Gaussian $R$-algebra. Therefore $c(f g h)=c(f) c(g) c(h) \subseteq Q$. Since $Q$ is a special 2-absorbing primary ideal of $R$, Theorem 7 implies that either $c(f) c(g)=c(f g) \subseteq Q$ or $c(f) c(h)=c(f h) \subseteq Q$ or $c(g) c(h)=c(g h) \subseteq \sqrt{Q}$. So $f g \in c(f g) T \subseteq Q T$ or $f h \in c(f h) T \subseteq Q T$ or $g h \in c(g h) T \subseteq \sqrt{Q T} \subseteq \sqrt{Q T}$. Consequently $Q T$ is a special 2-absorbing primary ideal of $T$.

$(\Leftarrow)$ Note that since $T$ is a content $R$-algebra, $Q T \cap R=Q$ for every ideal $Q$ of $R$. Now, apply Corollary 4(1).

The algebra of all polynomials over an arbitrary ring with an arbitrary number of indeterminates is an example of content algebras.

Corollary 10. Let $R$ be a Prüfer domain and $Q$ be an ideal of $R$. Then $Q$ is a special 2-absorbing primary ideal of $R$ if and only if $Q[X]$ is a special 2-absorbing primary ideal of $R[X]$.

Corollary 11. Let $S$ be a multiplicatively closed subset of $R$ and $Q$ be a proper ideal of $R$. Then the following conditions hold:

1) If $Q$ is a special 2-absorbing primary ideal of $R$ such that $Q \cap S=\varnothing$, then $S^{-1} Q$ is a special 2-absorbing primary ideal of $S^{-1} R$ with $2-\operatorname{ord}\left(S^{-1} Q\right) \leqslant 2-\operatorname{ord}(Q)$. 
2) If $S^{-1} Q$ is a special 2-absorbing primary ideal of $S^{-1} R$ and $S \cap$ $Z_{Q}(R)=\varnothing$, then $Q$ is a special 2-absorbing primary ideal of $R$ with $2-\operatorname{ord}(Q) \leqslant 2-\operatorname{ord}\left(S^{-1} Q\right)$.

Proof. By Proposition 11.

In view of Theorem 5 and its proof, we have the following result.

Corollary 12. Let $R=R_{1} \times R_{2}$, where $R_{1}$ and $R_{2}$ are rings with $1 \neq 0$. Let $Q$ be a proper ideal of $R$. Then the following conditions are equivalent:

1) $Q$ is a special 2-absorbing primary ideal of $R$;

2) Either $Q=Q_{1} \times R_{2}$ for some special 2-absorbing primary ideal $Q_{1}$ of $R_{1}$ or $Q=R_{1} \times Q_{2}$ for some special 2-absorbing primary ideal $Q_{2}$ of $R_{2}$ or $Q=Q_{1} \times Q_{2}$ for some prime ideal $Q_{1}$ of $R_{1}$ and some prime ideal $Q_{2}$ of $R_{2}$.

Corollary 13. Let $R=R_{1} \times R_{2}$, where $R_{1}$ and $R_{2}$ are rings with $1 \neq 0$. Suppose that $Q_{1}$ is a proper ideal of $R_{1}$ and $Q_{2}$ is a proper ideal of $R_{2}$. Then $Q_{1} \times Q_{2}$ is a special 2-absorbing primary ideal of $R$ if and only if it is a 2-absorbing ideal of $R$.

Proof. See Corollary 12 and apply [1, Theorem 4.7].

Corollary 14. Let $R=R_{1} \times R_{2} \times \cdots \times R_{n}$, where $2 \leqslant n<\infty$, and $R_{1}, R_{2}, \ldots, R_{n}$ are rings with $1 \neq 0$. For a proper ideal $Q$ of $R$ the following conditions are equivalent:

1) $Q$ is a special 2-absorbing primary ideal of $R$.

2) Either $Q=\times_{t=1}^{n} Q_{t}$ such that for some $k \in\{1,2, \ldots, n\}, Q_{k}$ is a special 2-absorbing primary ideal of $R_{k}$, and $Q_{t}=R_{t}$ for every $t \in\{1,2, \ldots, n\} \backslash\{k\}$ or $Q=\times_{t=1}^{n} Q_{t}$ such that for some $k, m \in$ $\{1,2, \ldots, n\}, Q_{k}$ is a prime ideal of $R_{k}, Q_{m}$ is a prime ideal of $R_{m}$, and $Q_{t}=R_{t}$ for every $t \in\{1,2, \ldots, n\} \backslash\{k, m\}$.

Proof. By Theorem 6.

\section{References}

[1] D. F. Anderson and A. Badawi, On $n$-absorbing ideals of commutative rings, Comm. Algebra 39 (2011) 1646-1672.

[2] D. D. Anderson, K. R. Knopp and R. L. Lewin, Ideals generated by powers of elements, Bull. Austral. Math. Soc., 49 (1994) 373-376.

[3] A. Badawi, On 2-absorbing ideals of commutative rings, Bull. Austral. Math. Soc., 75 (2007), 417-429. 
[4] A. Badawi, Ü. Tekir and E. Yetkin, On 2-absorbing primary ideals in commutative rings, Bull. Korean Math. Soc., 51 (4) (2014), 1163-1173.

[5] A. Badawi and A. Yousefian Darani, On weakly 2-absorbing ideals of commutative rings, Houston J. Math., 39 (2013), 441-452.

[6] J. A. Cox and A. J. Hetzel, Uniformly primary ideals, J. Pure Appl. Algebra, 212 (2008), 1-8.

[7] M. Hochster, Criteria for equality of ordinary and symbolic powers of primes, Math. Z. 133 (1973) 53-65.

[8] J. Hukaba, Commutative rings with zero divisors, Marcel Dekker, Inc., New York, 1988.

[9] H. Mostafanasab, E. Yetkin, U. Tekir and A. Yousefian Darani, On 2-absorbing primary submodules of modules over commutative rings, An. Şt. Univ. Ovidius Constanta, (in press)

[10] P. Nasehpour, On the Anderson-Badawi $\omega_{R[X]}(I[X])=\omega_{R}(I)$ conjecture, arXiv:1401.0459, (2014).

[11] D. G. Northcott, A generalization of a theorem on the content of polynomials, Proc. Cambridge Phil. Soc., 55 (1959), 282-288.

[12] J. Ohm and D. E. Rush, Content modules and algebras, Math. Scand., 31 (1972), $49-68$.

[13] D. E. Rush, Content algebras, Canad. Math. Bull., 21 (3) (1978), 329-334.

[14] R.Y. Sharp, Steps in commutative algebra, Second edition, Cambridge University Press, Cambridge, 2000.

[15] A. Yousefian Darani and F. Soheilnia, 2-absorbing and weakly 2-absorbing submoduels, Thai J. Math. 9(3) (2011) 577-584.

[16] A. Yousefian Darani and F. Soheilnia, On n-absorbing submodules, Math. Comm., 17 (2012), 547-557.

Hojjat

Mostafanasab

Ünsal Tekir

Gülşen Ulucak

\section{CONTACT INFORMATION}

Department of Mathematics and Applications, University of Mohaghegh Ardabili, P.O. Box 179, Ardabil, Iran E-Mail(s): h.mostafanasab@gmail.com

Department of Mathematics, Faculty of Science and Arts, Marmara University 34722, Istanbul, Turkey

E-Mail(s): utekir@marmara.edu.tr

Department of Mathematics, Gebze Technical

University, P. K. 14141400 Gebze, Kocaeli, Turkey

E-Mail(s): gulsenulucak@gtu.edu.tr

Received by the editors: 10.06.2017. 